\title{
Valorisation du sable issu des dragages d'entretien pour la lutte contre l'érosion côtière : le cas du Port de Dunkerque
}

\author{
Adrien CARTIER ${ }^{1}$, Antoine TRESCA ${ }^{2}$, Nicolas FORAIN ${ }^{2}$, \\ Gwenaëlle COTONNEC ${ }^{2}$, Arnaud HEQUETTE ${ }^{3}$
}

\section{GEODUNES, 59240 Dunkerque, France.} cartier@geodunes.fr

2. Grand Port Maritime de Dunkerque, Port 2505, 2505 Route de l'écluse Trystram, 59140 Dunkerque, France.

atresca@portdedunkerque.fr; nforain@portdedunkerque.fr;

gcotonnec@portdedunkerque.fr

3. Laboratoire d'Océanologie et de Géosciences, UMR CNRS 8187,

Université du Littoral Côte d'Opale, 32 Ave Foch, 62930 Wimereux, France.

arnaud.hequette@univ-littoral.fr

\section{Résumé :}

Le Grand Port Maritime de Dunkerque effectue de manière régulière des dragages d'entretien pour maintenir les tirants d'eau dans ses chenaux d'accès et bassins. Pour cela, il possède un arrêté préfectoral l'autorisant à draguer jusqu'à $2 \mathrm{Mm}^{3}$ de sable par an, auxquels peuvent s'ajouter ponctuellement des volumes issus de travaux neufs. Sa façade maritime de $17 \mathrm{~km}$ de long est délimitée par deux avant-ports qui perturbent significativement la dynamique hydrosédimentaire de ce secteur. La dérive littorale orientée ouest-est sur la façade Mer du Nord du littoral des Hauts-de-France est stoppée au niveau des deux jetées délimitant l'emprise côtière du Port. Les suivis morphodynamiques réalisés régulièrement montrent une tendance érosive qui souligne la nécessité d'une protection du linéaire côtier. En 2019, le Port de Dunkerque a finalisé son plan de gestion du trait de côte permettant de planifier la valorisation des sédiments issus des dragages d'entretien afin de renforcer son linéaire côtier vis-à-vis de l'érosion.

Mots-clés :

Gestion des sédiments, Erosion côtière, Dragage, Dunkerque.

\section{Introduction}

Depuis 1929, le développement du Port de Dunkerque est progressivement venu contrarier la dynamique hydrosédimentaire de la façade maritime locale. En effet, les flux côtiers de sédiments circulant d'ouest en est sont aujourd'hui stoppés par les avant-ports (voir Figure 1). Cet état de fait a été mis en exergue par le Plan Littoral d'Actions pour la Gestion de l'Erosion (PLAGE) (SMCO, 2003). Le PLAGE considérait dès le début des années 2000 que l'Unité de Gestion n 4 (UG4), délimitée par la jetée de St-Pol-sur-mer à l'est et les jetées du Clipon et du Ruytingen à l'ouest, était en érosion constante depuis 


\section{Thème 6 - Gestion durable des zones littorales et estuariennes}

de nombreuses années. Cette érosion s'est poursuivie ces dernières années (HEQUETTE et al., 2015), notamment au niveau de la digue du Braek, ouvrage majeur de la façade portuaire dans la protection contre les submersions marines.

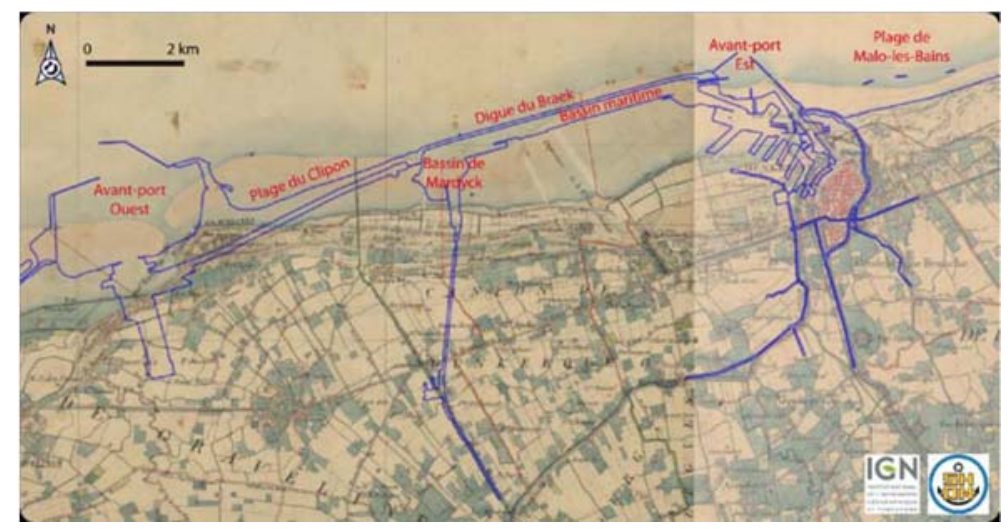

Figure 1. Superposition du trait de côte actuel à la carte d'Etat-Major (19e siècle)

(TRESCA, 2013).

Compte tenu des enjeux humains et économiques présents dans ce secteur, le maintien du trait de côte est une priorité absolue pour le port de Dunkerque, qui s'est doté d'un plan de gestion du trait de côte (PGTC), véritable outil de planification opérationnelle lui permettant de gérer efficacement sa façade littorale.

Le PGTC s'appuie principalement sur la valorisation du sable issu des dragages d'entretien des chenaux d'accès au port. Les opérations décrites par le PGTC consistent à réaliser des rechargements de plage sur des secteurs stratégiques et nécessitant une protection vis-à-vis de l'érosion côtière et/ou de la submersion marine.

\section{Cadre Réglementaire}

Le PGTC du Grand Port Maritime de Dunkerque considère et prend en compte les différentes directives Européennes (Directive Cadre sur l'Eau (DCE)), ainsi que les stratégies nationales (Grenelle de l'Environnement et de la Mer; Stratégie Nationale de Gestion Intégrée du Trait de Côte ; la Stratégie Nationale pour la Mer et le Littoral).

Le PGTC s'inscrit pleinement dans les différents plans régionaux déjà mis en place comme les PPRL, PPRI ; le Schéma Directeur d'Aménagement et de Gestion des Eaux ; le Schéma Régional de Cohérence Ecologique ; la Trame Verte et Bleue ; les sites Natura 2000. Enfin le PGTC est l'un des outils, au même titre que le Schéma Directeur de Dragage (SDD), de la mise en œuvre de la politique de développement durable de Dunkerque-Port déclinée au travers son Plan d'Aménagement et de Développement Durable (PA2D) (voir Figure 2) 


\section{XVİ̀mes Journées Nationales Génie Côtier - Génie Civil \\ Le Havre 2020}

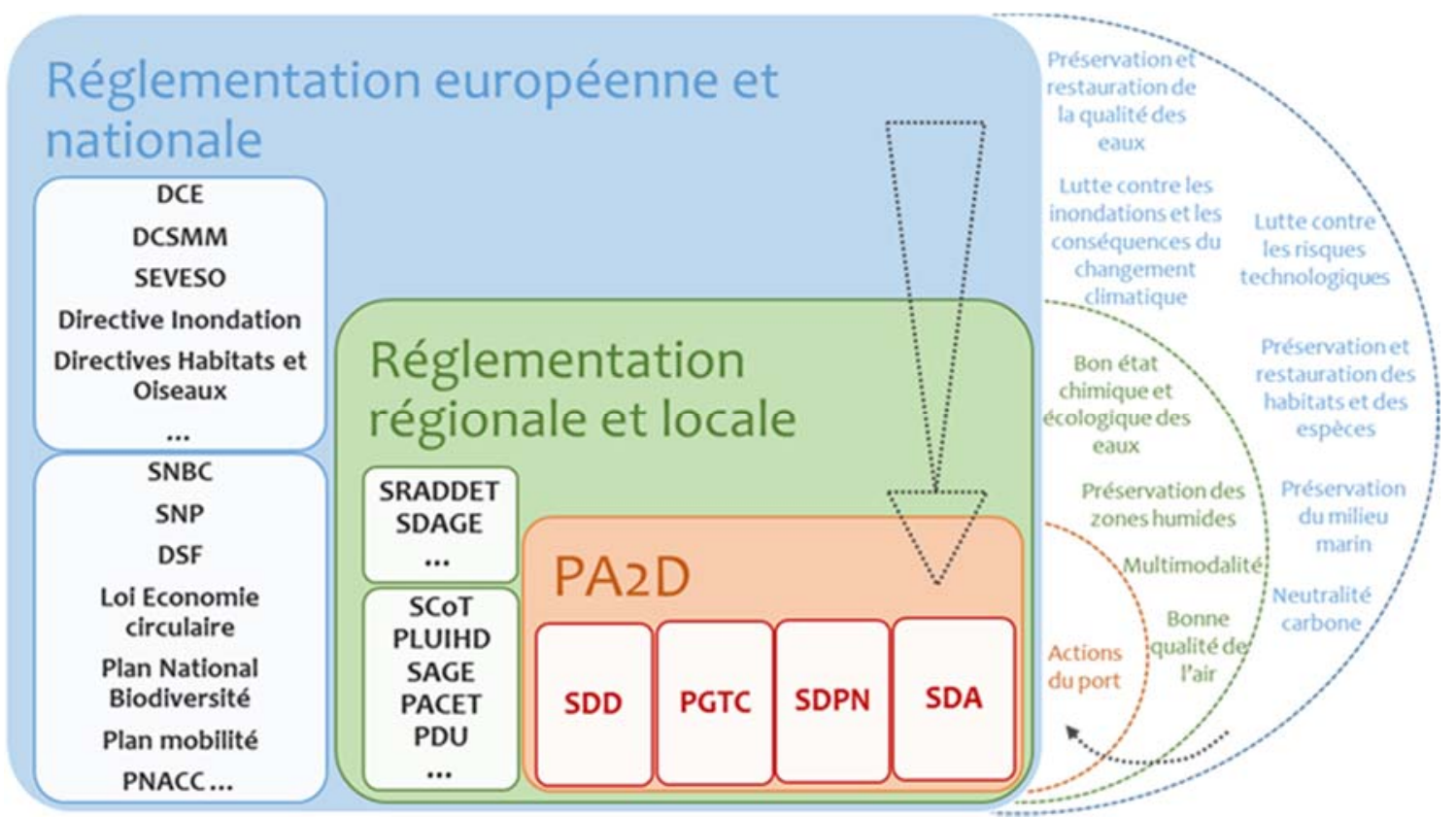

Figure 2. Organisation des principaux axes de développement de la politique environnementale de Dunkerque-Port (IDRA, 2015)

\section{Caractérisation de la façade maritime de Dunkerque-Port}

\subsection{Morphologie générale}

La façade maritime concernée par le PGTC est caractérisée par quatre bancs subtidaux sableux, les bancs de Snouw et du Braek qui délimitent l'UG4 au Nord, et plus près des côtes, les bancs de Mardyck et de St-Pol-sur-mer. Entre les bancs les plus au large et ceux près des côtes s'étend le chenal intermédiaire par lequel transitent les navires entre les deux avant-ports. Le chenal intermédiaire s'ouvre par ailleurs sur un chenal secondaire (naturel et non entretenu) séparant les bancs de Snouw et du Braek (voir Figure 3). La partie terrestre inclut plusieurs unités morphologiques comprenant les estrans, plages et cordons dunaires ainsi qu'une plateforme intertidale où deux flèches littorales se développent. La plateforme intertidale s'inscrit dans l'ensemble dit de la "Zone des Sternes". Des enrochements mis en œuvre dans le cadre de l'aménagement d'un terminal méthanier ont remplacé le cordon dunaire préexistant au Sud de la zone, alors qu'à l'ouest de l'anse, le cordon dunaire a subsisté, héritage d'une ancienne flèche littorale. Le long de la digue du Ruytingen et de la jetée du Clipon, l'estran accolé aux ouvrages correspond à la terminaison ouest du Banc de Mardyck. Si devant la jetée du Clipon, une fosse persiste, on retrouve aujourd'hui devant la digue du Ruytingen une plage totalement artificielle, issue des matériaux sableux extraits lors de l'aménagement d'un terminal méthanier. 


\section{Thème 6 - Gestion durable des zones littorales et estuariennes}

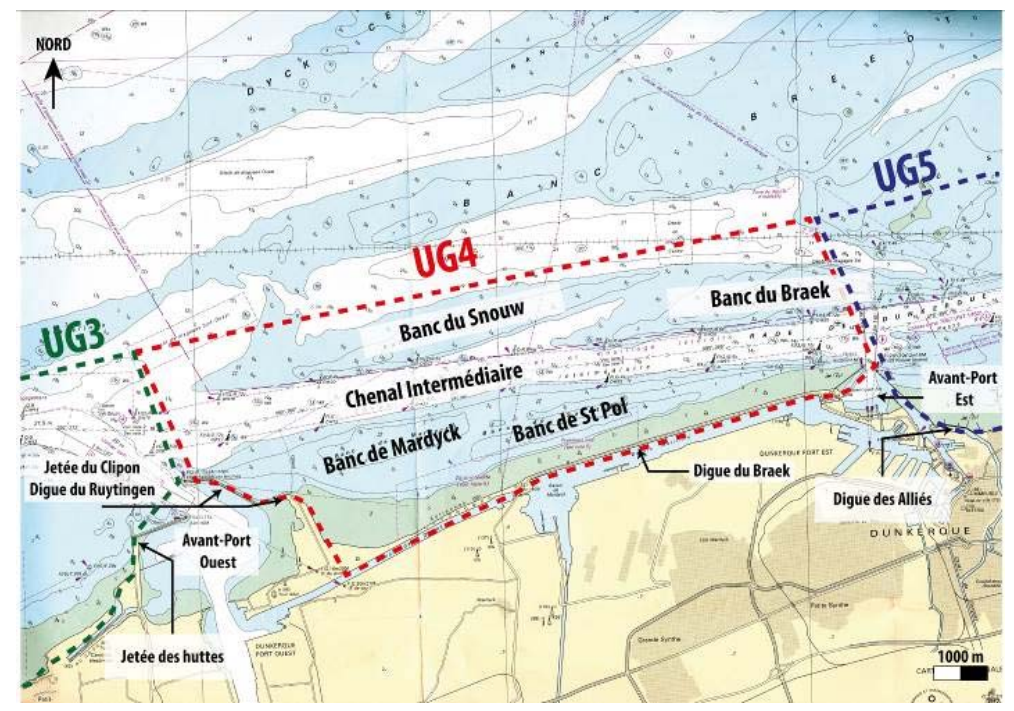

Figure 3. Délimitation des Unités de Gestion sur le territoire de Dunkerque-Port.

\subsection{Régime hydrosédimentaire}

La marée au niveau la façade maritime de Dunkerque-Port est caractérisée par un régime semi-diurne et par une houle à fetch court provenant surtout du secteur ouest. Les courants de marée présentent une forte dissymétrie, les courants de flot (vers ENE) étant nettement plus forts que les courants de jusant (vers OSO).

La granulométrie de la façade maritime est assez homogène, composée surtout de sables fins à moyens (grain entre 0,125 et $0,5 \mathrm{~mm}$ ) avec toutefois la présence de limons au niveau de la plateforme sablo-vaseuse

Le régime hydro-sédimentaire se traduit par un transport résultant orienté vers l'est. Les chenaux d'accès aux avant-ports est et ouest délimitant 1'UG4 constituent des pièges à sédiments. Les dragages d'entretien permettant d'y maintenir les tirants d'eau en extrayant le sable qui y sédimente (voir

Figure ).

Le transit littoral généré par les courants de houle est particulièrement intense le long de la digue du Ruytingen (60 $\left.000 \mathrm{~m}^{3} / \mathrm{an}\right)$. Le long des estrans, le transit augmente d'ouest en est, de $5000 \mathrm{~m}^{3} / \mathrm{an}$ à l'ouest de la plage du Clipon, il passe à $10000 \mathrm{~m}^{3} / \mathrm{an}$ devant la station Statoil et atteint $25000 \mathrm{~m}^{3} / \mathrm{an}$ à l'est de la Digue du Braek. Dans ce même secteur s'ajoute un transport d'environ $45000 \mathrm{~m}^{3} /$ an dans le bas estran, induit par les courants marins. Ainsi annuellement, $70000 \mathrm{~m}^{3}$ de sédiments se déposent aux abords de la jetée de St-Pol-sur-mer (DHI, 2015).

Dans le chenal intermédiaire, les courants de marée induisent un transport potentiel d'au moins $150000 \mathrm{~m}^{3} / \mathrm{an}$, portant vers l'est. Enfin, les dépôts dans le chenal intermédiaire en provenance du Banc de Mardyck sont estimés entre 75000 à $100000 \mathrm{~m}^{3} / \mathrm{an}$. 


\section{XVİ̀mes Journées Nationales Génie Côtier - Génie Civil \\ Le Havre 2020}

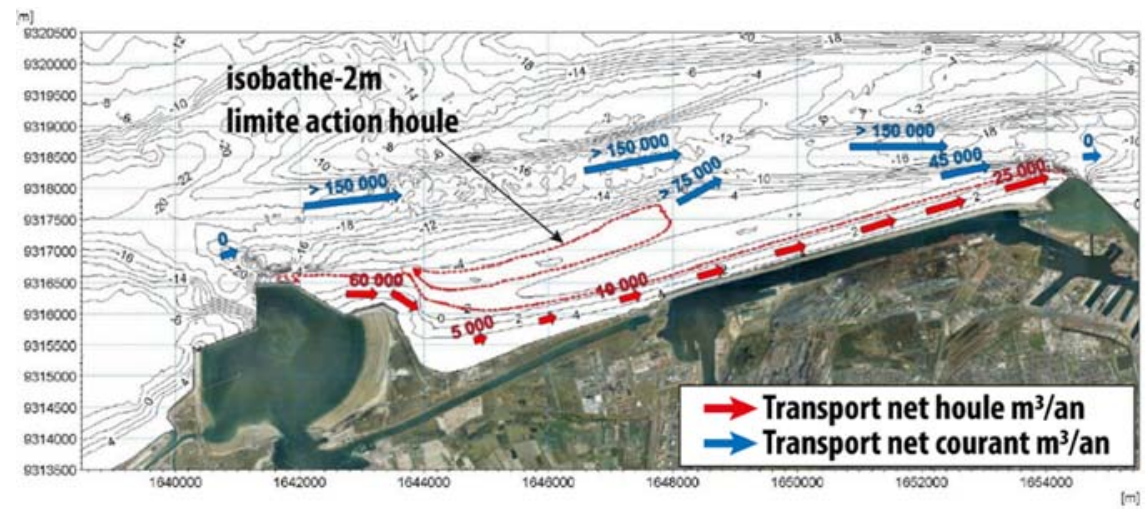

Figure 4. Synthèse du transport sédimentaire en m³/an, (DHI, 2015).

\subsection{Bilan sédimentaire}

Le bilan montre un déficit sédimentaire global d'environ $-9 \mathrm{Mm}^{3}$ sur les zones $\mathrm{A}, \mathrm{B}, \mathrm{D}$, F, G entre 2000 et 2014 (voir Figure ). Le secteur D caractérisant la plage et l'avant-plage de la digue du Braek est l'un des plus déficitaires avec une perte de $-4,7 \mathrm{Mm}^{3}$. Au sein du secteur B, globalement en accrétion, il convient de distinguer la zone du Clipon qui présente une érosion de $80000 \mathrm{~m}^{3}$, et un recul du cordon dunaire de $50 \mathrm{~m}$.

\section{Description des volumes de sédiment disponibles}

\subsection{Dragages d'entretien et grands travaux}

Les volumes extraits du chenal intermédiaire lors des dragages d'entretien peuvent varier d'une année à l'autre en fonction de la sédimentation. En outre, le port de Dunkerque a entamé il y a quelques années une phase de rattrapage progressive des cotes d'exploitation annoncées dans le permis d'immersion, et exigées par la Capitainerie du port afin d'accueillir les navires en toute sécurité. Ainsi, en 2014, $373000 \mathrm{~m}^{3}$ de sédiments ont été extraits (IDRA, 2015) et en 2015/2016, les dragages ont été bien plus conséquents $\left(925000 \mathrm{~m}^{3}\right)$ en raison d'une forte sédimentation dans le secteur C (voir figure 5).

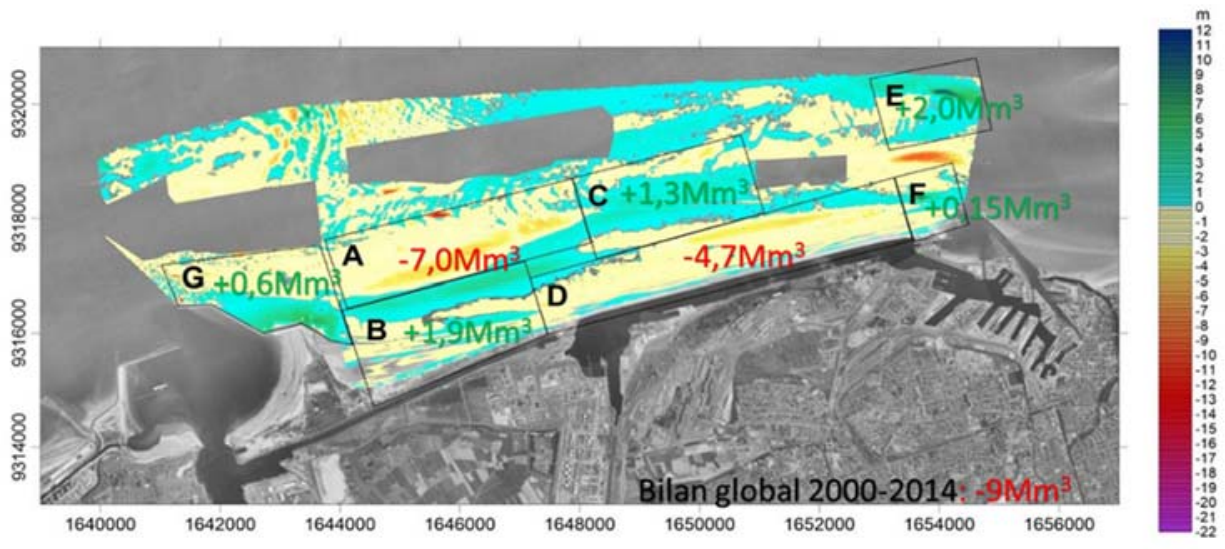

Figure 5. Bilan sédimentaire par secteur au sein de l'UG4 de 2000 à 2014. 


\section{Thème 6 - Gestion durable des zones littorales et estuariennes}

Selon DHI (2015), en "régime permanent" (sans rattrapage des cotes) et en l'état actuel des fonds, les volumes disponibles liés aux dragages d'entretien doivent s'établir aux alentours de $250000 \mathrm{~m}^{3}$ dans le chenal intermédiaire $\left(150000\right.$ à $200000 \mathrm{~m}^{3}$ en partie centrale (C) et 50000 à $100000 \mathrm{~m}^{3}$ aux abords de la jetée de St-Pol-sur-mer (F)).

Dans le cadre du projet d'extension Cap 2020, un volume d'environ $10 \mathrm{Mm}^{3}$ de sable pourrait résulter des travaux. La cote d'exploitation du chenal intermédiaire pourrait être également modifiée apportant un volume d'environ $1 \mathrm{Mm}^{3}$ de sable.

\subsection{Les opérations déjà réalisées}

Lors de la construction du terminal méthanier (2011), environ $4 \mathrm{Mm}^{3}$ de sable ont été extraits dont une partie a été valorisée par un rechargement de plage au droit de la digue du Ruytingen (GEODUNES, 2014 ; SPODAR, 2018) afin de protéger l'appontement des navires dans la darse du terminal.

En mars 2014, le GPMD, en tant que Maitre d'ouvrage délégué pour l'Etat, a entrepris de refouler 1,5 $\mathrm{Mm}^{3}$ au droit de la digue des Alliés, ouvrage clé dans la protection contre les submersions marines dans l'agglomération Dunkerquoise (40 000 habitants protégés) (CARTIER et al., 2014 ; SPODAR et al., 2018).

\section{Dernière opération réalisée : mise en place d'un by-pass}

\subsection{Description technique}

En 2019, en vue de faciliter les opérations de rechargement de plage à l'aide du sable des dragages d'entretien, le port de Dunkerque a entrepris la création d'un émissaire de refoulement, sorte de by-pass entre la zone à draguer dans le chenal intermédiaire de navigation et le secteur côtier en érosion.

La terminaison de la canalisation se fait par un tuyau flottant à laquelle la drague vient se connecter. La canalisation est mise en pression, et la mixture sable-eau se déverse sur la plage par refoulement hydraulique. Le sable est repris par des engins terrestres en vue de le répartir et reconstituer la pente de plage adéquate (voir Figure ).

Bien que cela représente un investissement initial conséquent, elle maximise les chances que le sable refoulé reste en place (contrairement au Rainbowing). La proximité du point de connexion vis-à-vis de la zone à draguer permet de réduire significativement les temps de cycle, créant ainsi une économie à chaque chargement.

\subsection{Evolution topographique}

Environ $1 \mathrm{Mm}^{3}$ de sédiments ont été refoulés fin 2019, rehaussant le niveau de la plage de 2 à $3 \mathrm{~m}$ sur la zone intertidale devant la digue du Braek. Les derniers relevés topographiques réalisés en janvier 2020 font état d'un bilan volumétrique de $+480000 \mathrm{~m}^{3}$ sur la zone d'emprise du rechargement suggérant qu'environ $50 \%$ du volume total refoulé 


\section{XVI èmes Journées Nationales Génie Côtier - Génie Civil \\ Le Havre 2020}

a été dispersé au cours des opérations (voir Figure ). Constat tout à fait cohérent lorsque les rechargements s'opèrent sur un estran bas soumis à de forts courants de marée.

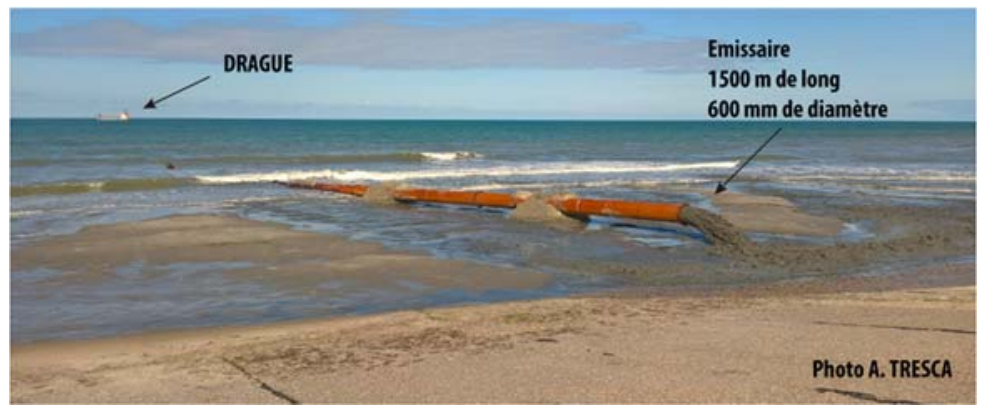

Figure 6. Illustration du rechargement par le by-pass installé en 2019.

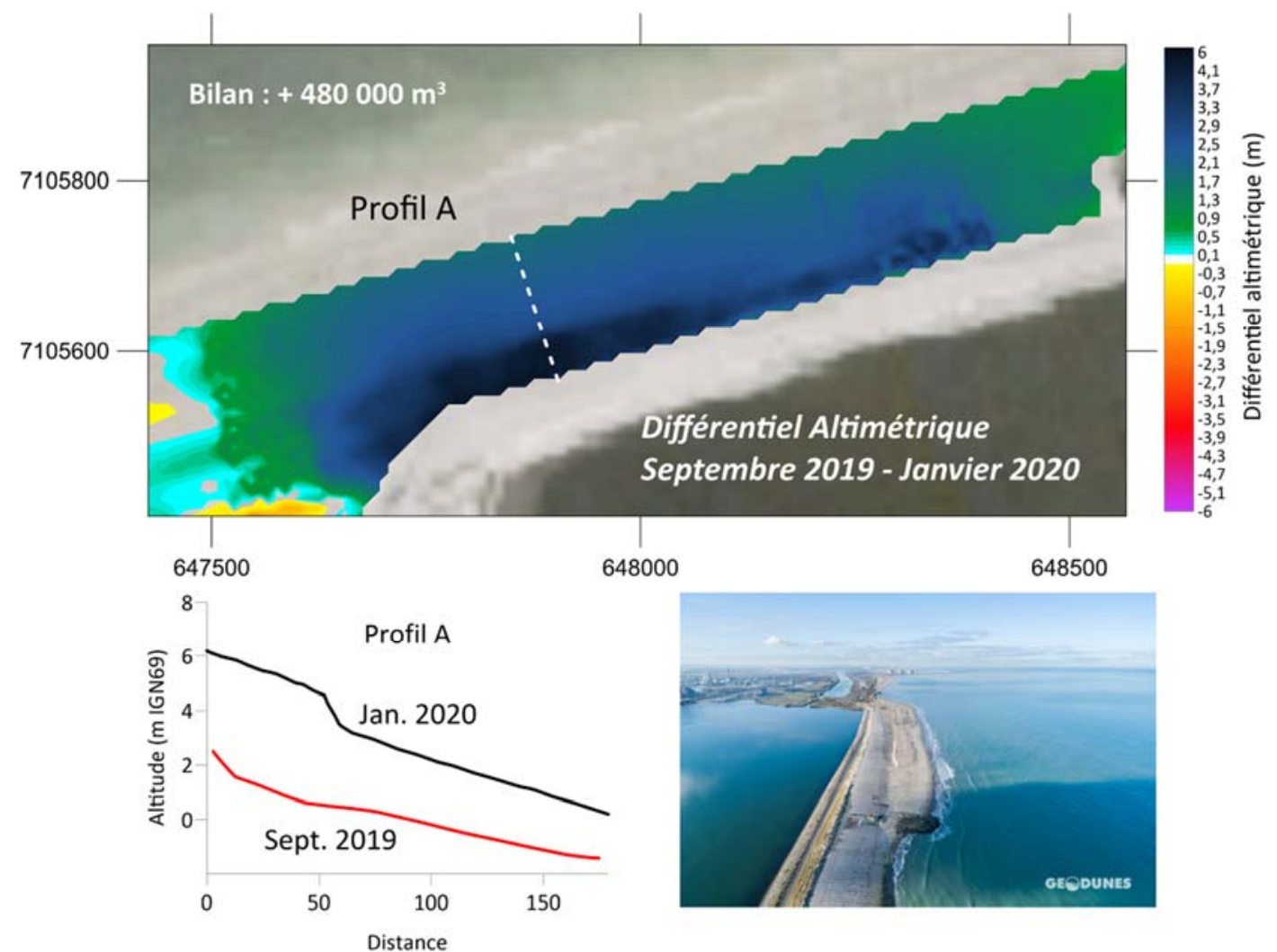

Figure 7. Rechargement de la plage de la digue du Braek fin octobre 2019.

\section{Conclusions}

Le plan de gestion du trait de côte du Grand Port Maritime de Dunkerque permet une approche holistique des différentes problématiques que connaît sa façade littorale. Des opérations de rechargements de bancs et de plages de grande envergure, à partir des sédiments des dragages d'entretien de l'UG4 ou de travaux neufs (agrandissements de bassins), sont envisagées pour compenser le phénomène d'érosion induit par le développement des infrastructures portuaires depuis le début du $20^{\text {ème }}$ siècle. Avec ce 


\section{Thème 6 - Gestion durable des zones littorales et estuariennes}

PGTC, l'objectif du port de Dunkerque est multiple : (i) Solutions souples permettant de protéger les infrastructures (digues); (ii) Ressource disponible via les dragages d'entretien avec restitution au système côtier; (iii) Maintien de la biodiversité par la conservation des habitats. Le PGTC prévoit aussi des mesures de gestion des sables éoliens pour favoriser le développement des dunes et limiter les envols sur les infrastructures portuaires. Un suivi pluridisciplinaire pendant et après les phases de travaux est indispensable en vue de disposer d'une vision intégrée de l'UG4. Cela doit pouvoir également permettre de faire évoluer le plan de gestion en mettant en lumière des interventions non prévues initialement : nouveaux secteurs côtiers vulnérables, impact spatial des rechargements sur la sédimentation.

\section{Références bibliographiques}

CARTIER A., TRESCA A., GRUNNET N., MICHARD B., FORAIN N., VIAL T. (2014). Confortement d'un ouvrage de prévention des inondations et des submersions marines : l'exemple de la digue des Alliés à Dunkerque. Journées Nationales Génie Côtier - Génie Civil, Dunkerque, pp 641-48. https://doi.org/10.5150/jngcgc.2014.070

DHI (2015). Rapport de synthèse de la tranche ferme : comportement morphodynamique du site d'étude. Rapport pour le compte de Dunkerque Port, $101 \mathrm{p}$.

GEODUNES (2014). Analyse de l'évolution morphodynamique du rechargement de la plage et de l'avant-côte au droit de la Digue du Ruytingen. Rapport pour le compte de Dunkerque Port, $47 \mathrm{p}$.

HEQUETTE A., CARTIER A., RUZ M.-H., MARIN D., SIPKA V. (2015). Suivi morpho-sédimentaire de l'UG4 - Bilan 2010-2014. Rapport du Laboratoire d'Océanologie et de Géosciences-Université du Littoral Côte d'Opale pour le Grand Port Maritime de Dunkerque, $331 \mathrm{p}$.

IDRA (2015). Plan de gestion opérationnelle des dragages du Grand Port Maritime de Dunkerque. Rapport pour le compte de Dunkerque Port, 75 p.

SMCO. (2003). Plan littoral d'action pour la gestion de l'érosion. Syndicat Mixte de la Côte d'Opale, $70 \mathrm{p}$.

SPODAR A. (2018). Analyse morphodynamique de rechargements sédimentaires sur le littoral du nord de la France: le cas de la façade portuaire de Dunkerque. Thèse de Doctorat, Université du Littoral Côte d'Opale, Wimereux, 368 p.

SPODAR A., HEQUETTE A., RUZ M-H., CARTIER A., GREGOIRE P., SIPKA V., FORAIN N. (2018). Evolution of a beach nourishment project using dredged sand from navigation channel, Dunkirk, Northern France. Journal of Coastal Conservation 22, $\mathrm{n}^{\mathrm{o}} 3$, pp 457-74. https://doi.org/10.1007/s11852-017-0514-8

TRESCA A. (2013). Contrôle souple de la dynamique éolienne sur un littoral artificialisé et propositions de gestion : le cas de la façade maritime du grand port de Dunkerque. Thèse de doctorat, Université du Littoral Côte d'Opale, Dunkerque, 398 p. 